\title{
Loss of Epistemic Self-Determination in the Anthropocene
}

\section{Ian Werkheiser}

To cite this article: Ian Werkheiser (2017): Loss of Epistemic Self-Determination in the Anthropocene, Ethics, Policy \& Environment, DOI: 10.1080/21550085.2017.1342966

To link to this article: http://dx.doi.org/10.1080/21550085.2017.1342966

曲 Published online: 28 Jun 2017.

Submit your article to this journal

Q View related articles ¿

View Crossmark data \lceil 


\title{
Loss of Epistemic Self-Determination in the Anthropocene
}

\author{
Ian Werkheiser \\ Department of Philosophy, University of Texas Rio Grande Valley, TX, USA
}

\begin{abstract}
One serious harm facing communities in the Anthropocene is epistemic loss. This is increasingly recognized as a harm in international policy discourses around adaptation to climate change. Epistemic loss is typically conceived of as the loss of a corpus of knowledge, or less commonly, as the further loss of epistemic methodologies. In what follows, I argue that epistemic loss also can involve the loss of epistemic self-determination, and that this framework can help to usefully examine adaptation policies.
\end{abstract}

\section{Introduction}

Adaptation to the Anthropocene is essential, even assuming partial success for remediation and mitigation schemes to address the various anthropogenically driven environmental changes which have already begun. These adaptations will in part be physical, as we make changes to our infrastructure as well as purposive changes to our environment, in response to the accidental changes we have made until now. Adaptations will also in part be sociopolitical, as we must adapt our institutions to the conditions of this new era in which human effects on the environment are substantial enough to overwhelm previously well-established patterns. In what follows, I will focus on this second kind of adaptation. Social adaptation could be in the direction of increased centralization, hierarchy, and coercion in order to survive on an increasingly dangerous planet. It could also be in the direction of decentralization, self-organization, and solidarity. I believe that the second of these courses is often the more just, but I also believe independently that the latter direction holds out more promise for successful, resilient adaptation to anthropogenic risks. This has strong implications for how we ought to evaluate possible adaptation strategies and how we ought to approach climate loss and damage.

One important issue to think through when addressing adaptation to the Anthropocene is harm. This is because if key changes to the environment in this new epoch are anthropogenic (as the name implies), then these harms are potentially ones for which people are culpable. Some potential harms may be reduced or perhaps avoided entirely, while other harms may be largely unavoidable. An awareness of the probability, avoidability, and seriousness of various harms can inform the difficult triage of adaptation societies will face. This is true for everyone; however, harms take on a special importance for marginalized groups 
and rightly provoke special consideration in international discussions of managing the Anthropocene. Given that many harms will be suffered by those with the fewest resources (who are also among the least responsible for the causes of those anthropogenic harms), some argue on both justice and charity grounds for wealthier societies to help them minimize the avoidable harms through adaptation, and to compensate them for the unavoidable harms (E.g. Baatz, 2013; Mendelsohn, Dinar, \&Williams, 2006). These two forms of aid reinforce each other, as the money for compensation is a promising resource for marginalized societies to use in adaptation. Such aid is also arguably in the best interests of the societies providing it, given that sufficient harms to low-resource communities and nations are likely to result in massive migration into those wealthier societies, where assistance is likely to be much more costly than it would have been for communities in their original location (See, e.g. Biermann \& Boas, 2010). The recognition of the need for wealthier societies helping poorer ones with adaptation and compensation for harms is now very present within the discourse around climate change, ${ }^{1}$ and many people are considering what this should look like in international policy discussions, such as those around the Warsaw International Mechanism for Loss and Damage Associated with Climate Change Impacts (WIM). ${ }^{2}$

There are many potential harms in the Anthropocene, including economic losses, cultural losses, harms to health and well-being, damage, and more besides. While these are all important, I will focus on the harms of loss and damage. Specifically, I will focus on epistemic loss and damage. Epistemic loss usually refers to the loss of both knowledge and knowledgerelated practices, and is already present in international discourse around adaptation (E.g. Alessa, Kliskey, \& Williams, 2010). In addition to those losses already acknowledged in this discourse, there is another possible way of understanding one kind of epistemic loss and damage, namely as damage to and the loss of epistemic self-determination.

I will briefly define epistemic self-determination and argue for its importance to communities, both as an end in itself and as a means to other goods. I will particularly highlight the ways in which epistemic self-determination allows communities to function in less centralized cooperation with one-another, while a loss of epistemic self-determination usually leads to centralization and epistemic dependence. I will then argue that a loss of epistemic self-determination for many marginalized communities is a probable harm arising from some of the changes in the Anthropocene, and one which should be avoided when possible and compensated for when it is unavoidable. I will also discuss how some attempts at adaptation themselves can develop or undermine epistemic self-determination for those marginalized communities. Ultimately, I argue that damage to and even the loss of epistemic self-determination is a serious harm for marginalized communities, and that this damage and loss ought to be addressed in international policy.

\section{Epistemic Self-Determination}

One of the recognized potential harms of the Anthropocene is epistemic loss. There is growing awareness of the loss of 'indigenous/local knowledge' in climate change (UNFCCC Technical Paper 2, 2013, p. 4), and this can be easily extended to other anthropogenically driven environmental change, from species extinction through overfishing, to fertilization run-off, to light pollution, and so (depressingly) on. This loss of knowledge is often viewed from the perspective of a loss of a corpus, and the harms associated with it include a loss of something a community values, and a harm to cultural or community identity (Figueroa, 
2006). On the other hand, this epistemic loss is also sometimes viewed from the perspective of a loss of methodologies and epistemic practices rather than knowledge, and the harms associated with it include harm to the epistemic agents' ability to adapt and survive (Chief, Daigle, Lynn, \& Whyte, 2014). The harms and losses from both perspectives are real and important to address in a time of anthropogenic environmental harm, particularly for those cultures (such as many indigenous cultures) that recognize their values, identity, plans, and knowledge as mutually supporting each other (e.g. Voggesser, Lynn, Daigle, Lake, \& Ranco, 2013; Wildcat, 2013). There is another possible perspective on knowledge loss, one that has not been sufficiently examined in the discourse, but which is a useful way to understand some of the harms associated with epistemic loss, and some of the strategies we might employ to minimize those harms. This perspective is the loss of epistemic self-determination, the ability of community members to jointly determine and engage in the epistemic practices of their community, which can include methodologies for gaining knowledge as well as evaluative assumptions for accepting or rejecting knowledge. ${ }^{3}$ While this term is fairly rare in common discourse around the Anthropocene, there are several reasons to think that as a concept it is important enough that those engaged in climate policy ought to value it and see its loss as a serious harm.

The first reason to value epistemic self-determination is that it can be viewed as a subset of general self-determination, in the way that term is used in transnational social-justice contexts, such as in the environmental justice and food sovereignty movements. In this discourse, self-determination is seen as included within democracy. As Carol Gould says, people have a prima facie right in a democracy to determine their own activities. Many activities which are important to people can only be achieved in a collectivity with others, and thus no individual has a prima facie right to determine those shared activities more than any other participant. These community freedoms are goods in themselves in this discourse (e.g. Carter, 1999, p. 248) as goals which are valued and pursued by people via their communities. They are also instrumentally vital for developing many individual freedoms (e.g. Evans, 2002; Ibrahim, 2006). They are also partly constitutive of what democracy is; democratic pursuit of shared goals by communities of people is what Gould calls self-determination by that community (Gould, 2014, pp. 87-89). As she argues, this sense of self-determination comes with a duty by social and political organizations to support it:

[People's] development of capacities, building of relationships, and fulfillment of long-term goals depends both on their own choices, individual or joint, and on the availability of the means for their freedom to become effective, where several of these means are specified in human rights. We can rightly be critical when these prerequisites are not provided through social and political organization, which instead should aim to fulfill them. (Gould, 2014, p. 74)

In the context of environmental justice activism and scholarship, a good example of work incorporating self-determination is Taylor's overview (2000) of the creation of environmental justice as a paradigm. There, she stresses the importance of self-determination to the movement, which she defines primarily as 'The rights of people of color to determine their own political, economic, and cultural futures'but says that self-determination also involves'reflection and self-healing.' (Taylor, 2000, p. 542). Taylor argues that because the environmental experiences of people of color are quite different than the experiences of whites, there was a redefinition of environmental activism by people of color to have three new components: 'autonomy or self-determination, land rights, and civil or human rights.' (Taylor, 2000, p. 533). The different environmental experiences of people of color relevant to self-determination 
are that 'People of color had little or no choice about where they lived, what jobs they did, or how they interacted with the land.' (Taylor, 2000, p. 534). As a result, 'One of the enduring struggles of people of color is that of self-determination-the struggle to define who they are and how they interact with the land.' (Taylor, 2000, p. 534).

In the context of food sovereignty activism and scholarship, a good example of work incorporating self-determination is Menser's description (2008) of the food sovereignty organization La via Campesina. Menser says that La via Campesina as an organization pursues what he calls'MaxD,' meaning maximally democratic participation. Menser defines MaxD as pursuing '(i) democratic self-determination; (ii) capacity development for individuals and groups; (iii) delivery of economic, social, and/or political benefits; and (iv) the construction, cultivation, proliferation, and interconnection of movements and organizations that embody the first three tenets.' (Menser, 2008, p. 24). This conception of self-determination is one which is both focused on the development of the community and its members, and just relationship both internally and externally.

Self-determination in the sense it is used in this discourse is very important for everyone in order for individuals to flourish through (in part) association with others in just communities and the pursuit of communal goals and projects. It is also of particular importance for marginalized or oppressed communities, who are denied the full expression of their identities as members of communities and to pursue communal projects. This is an important justice issue, and the loss of self-determination is a serious harm, particularly for communities that do not see their values and goals well represented in larger social institutions. This includes those self-determined goals and projects around epistemic practices, such as gaining knowledge, evaluating the knowledge of others, maintaining that knowledge, adapting the knowledge to changing situations, and continuing the knowledge within the community into future generations (see the example of the Karuk people below) (Werkheiser, 2015). Epistemic self-determination has been under-examined by those working on developing frameworks for loss and damage, and this is unfortunate because without the ability for a community to evaluate decisions and information via methodologies that they understand and accept, and which incorporate their values, other forms of self-determination are much more difficult.

The second reason to value epistemic self-determination is the ways in which it facilitates communities' participation in decision-making processes. For participation in policy decision-making to be meaningful, it is necessary that there be at least a range of different alternative policies which could be the outcome of these processes, and at least some of those policy outcomes should reflect the values, knowledge (including evaluative assumptions and epistemic methodologies as well as data), and goals of the participants (Werkheiser, 2015). Many aspects of these meaningful alternatives are epistemic. This is true both in the sense that the alternative policies should include the knowledge and methodologies of the participants, and also because in order to include the goals and values of the participants, it is necessary to have the epistemic capacities to know or learn what those goals and values are.

Though all this is true for individuals participating in a decision-making process, it is more useful to look at the level of communities participating in a given process, for several reasons. In part, this is because complex methodologies for gaining knowledge and evaluating evidence are not the sort of thing held by individuals, but rather by communities. This is the case for scientific knowledge, as well as for the folk knowledge and traditional knowledge 
of communities. Also in part, this is because at least some people see their communities as the site of participatory injustice when the community is not consulted in decisions affecting it, even if many individuals within the community are consulted. ${ }^{4}$

Consulting a community requires allowing the community to engage in its own internal deliberation processes, something which does not occur when surveying members of a community. This difference matters for a host of reasons. One is that members of the community may want to change their opinion when they hear how other members of their community feel and would like this opportunity before being consulted, or would at least want to maintain community relations by listening to fellow members before participating in a decision affecting them all. Another reason is that for many communities, members have a strong interest in making that community (qua community) act rationally and consistently, and so must take other members' opinions into account to make a joint decision (Pettit, 2001). This is most obvious when the community is a formal entity, particularly one with deferred decision-making (polling people in a country isn't the same as engaging in diplomacy with that country) but similar considerations can come up in groups like families and clubs. Thus, an important (but often underexamined) scale for thinking about participatory justice in policy decision-making is marginalized and oppressed communities.

A recent example of environmental injustice against a community (in addition to individuals) is the Dakota Public Access Pipeline (DAPL). As Kyle Whyte, a philosopher and Potawatomi scholar-activist, has argued, the harms of DAPL are best viewed as an injustice of settler colonialism against the tribe. The injustices that were part of this include the attempt by the settler colonialist society to erase the tribe through taking land, disrupting the environment until traditional economic and cultural relations between the tribe and various species that are important to it become impossible, and a host of other harms. Note that this erasure could happen without the death of any individuals in the tribe (in actual practice there has been great violence at DAPL protests which may yet result in deaths, and the pipeline once completed could well lead to deaths). Another injustice according to Whyte is a failure to respect the self-determination and self-governance of the tribe through insufficient consultation with and participation from the tribe in the decisions leading up to approving DAPL (Whyte, 2017).

Some have argued that for disadvantaged communities who cannot easily generate their own alternative policies for any number of possible reasons including a lack of viable options or the community capacities to do so, a burden to generate alternatives which embed the vulnerable community's values, knowledge, and goals falls on dominant societal institutions (Shrader-Frechette, 1991, p. 207). However, there are several reasons to think that these alternatives are suboptimal for those communities. First, as argued above, self-determination is very important to many individuals and communities, and outsourcing the generation of alternative policies does not promote that good. Second, there is the risk that external experts would mis-recognize the values and other aspects of the community. Third, such a process can further weaken epistemic self-determination as the community is rendered epistemically dependent on external expertise, which may be withdrawn, and which may be susceptible to conflicts of interest, such as might arise from the funding source external experts are using to work on behalf of the communities. Fourth, the community is less likely to be able to advocate for and defend their preferred alternatives if they were generated by an external process, and less likely to be able to modify them in ways which preserve the elements that are most important to them. For example, in food policy, some policies 
on-balance promote epistemic self-determination, such as the promotion of local food policy councils and local food hubs. Neither of these policies gives full license to communities to self-determine their own food systems regardless of the effects on other communities, but they do empower those communities to participate in decisions around their food systems and to gain epistemic capacities to learn more and make better decisions in the future. Other food policies undermine epistemic self-determination (though they may achieve other worthwhile ends), such as the 'dumping' of food on countries suffering famine without considering the effect on local food producers or local consumption patterns. If continued for a long enough time, such policies can harm a community's ability to retain traditional knowledge around food production and preparation (Werkheiser, 2016).

With this understanding of the importance of epistemic self-determination in place as a good valued and pursued in itself by many people, as an important element of meaningful participation in important policy decisions, and as partly constitutive of democratic processes, we can look at how it is likely to be affected in the Anthropocene by changes to the world in which these communities live, as well as by various attempts to adapt. This can provide a framework for evaluating possible adaptation policies by whether they increase and utilize epistemic self-determination for communities or undermine it.

\section{Epistemic Self-Determination in the Anthropocene}

In the Anthropocene, epistemic self-determination is under threat from various directions. One threat comes from engagement with scientific experts who are external to the community. In an era of rapid changes from human activity which are quite difficult to predict, traditional knowledges (e.g. of local ecosystems) face novel challenges as they are rendered less useful or even misleading. Further, what prediction is possible requires an often unprecedented knowledge of complex social systems (e.g. predicting future climate change requires understanding the efficacy as well as the political viability of international mitigation proposals). When this happens, traditional knowledge must be supplemented by new research, which can often benefit from engagement with science communities, including those external to the communities' traditional networks (Whyte, in press). However, this engagement can sometimes provide local communities with recommendations based on methodologies they don't understand, motivated by unknown values. This can be an erosion or in extreme circumstances an outright abrogation of the community's epistemic self-determination.

This need not always be the case. It is also possible for adaptation to at least sometimes not erode and indeed to increase epistemic self-determination for communities, and doing so would not only achieve the goods described above, but would also make for a more resilient adaptation, in that the adaptation is more likely to be modifiable when necessary to match external requirements (e.g. Werkhseiser, 2014). This can be done in part through open communication about external expert methodologies and their assumptions, and providing what resources communities need (time, money, access to those experts) to critically evaluate both the conclusions and the methodologies to see how well they work in a particular community's contexts. This can allow communities the ability to both have their practices better meet external situations, and provide them with better methodologies to continually reevaluate and readapt those practices moving forward (e.g. Norton, 2005).

A good example of the perils and promises of adaptation to the Anthropocene on epistemic self-determination, as well as the importance of respecting and supporting epistemic 
self-determination in any project with an adaptation component, can be found by looking at the situation of the Karuk people of the Klamath River area of the Pacific Northwest of the United States, primarily Northern California. The Karuk tribe faces severe problems of community sovereignty and viability (See, e.g. Werkhseiser, 2014). Some of these are social and political, as when their traditional food practices are curtailed, illegalized, and/or made impossible. Dams are exterminating salmon runs. Hunting game and fishing are strictly regulated by wardens, leading to altered hunting patterns (at night, in secret, and alone), the arrest of many Karuk people, and a great reduction in food from hunting. Karuk people also do not have access to their land which now 'belongs' to private owners or state parks, making foraging for mushrooms, acorns, and other foods impossible to do in sufficient quantities to supplement their diet, and illegalizing many traditional practices to increase the fertility of their land such as burning. These problems are exacerbated by anthropogenic change, as droughts make the community far more vulnerable to wildfires and even less able to fish from dwindling streams (Karuk Tribe of California, 2006, n.d.; Norgaard, 2004; Pierce, 1998; Reed \& Norgaard, 2010).

Attempts by authorities to alleviate these problems in response to activism have not been taken with an eye toward epistemic self-determination for this community. For example, the US Government and the California and Oregon State governments have given food aid in the form of free canned goods, cereals, and the like directly to the Karuk people, and indirect aid via food stamps and other food aid programs. Thus far, the outcomes from these programs have not been positive. Many Karuk people feel that their culture and way of life are being eroded by their inability to obtain the kind of food in the manner that has been co-constituted with their culture, and they fear the loss of knowledge around their traditional practices (Karuk Tribe of California, 2007, n.d.; Pierce, 1998; Reed \& Norgaard, 2010). The provision of food and supplies in this manner is also leading to adverse outcomes even by the programs' own lights. Forty-two percent of Karuk respondents living in the Klamath River area are 'food insecure' or 'hungry' despite relying on food assistance (Norgaard, 2004). At the same time, they are also suffering from obesity and diabetes (Reed \& Norgaard, 2010). Poverty rates are nearly three times the US average (Norgaard, 2004).

To address these issues, a number of projects which support rather than undercut traditional knowledge are being pursued. These projects seek to 'Explore when, where and how Karuk TEK [Traditional Ecological Knowledge] can be integrated into research and thereby landscape level conservation' (Reed, Sarna, Diver, \& Lynn, 2012) with a particular focus on food species like salmon and acorns, as well as making the landscapes more fire resilient. This project and others like it have the potential to do several useful things for the Karuk. They could help preserve traditional knowledge by valuing it in an official context, support traditional knowledge by providing insights from modern adaptive management practices, make it easier to implement traditional knowledge on the landscape by incorporating some of the insights of traditional knowledge into management plans, and hopefully make the landscape from which Karuk get much of their traditional foods more resilient to the increasingly frequent wildfires that are predicted in a climate change model (Karuk Tribe of California, 2007). Most importantly for our purposes, because this traditional knowledge incorporates the knowledge, methodologies, and epistemic values of the community, and is a product of community-based projects and goals, it increases their epistemic self-determination.

Another important threat to communities' epistemic self-determination in the Anthropocene is from political and economic experts who are external to the community. 
If the preceding discussion addressed threats to epistemic self-determination around the ecological side of the socio-ecological systems in which communities are embedded, a new threat comes from the changing social side of that complex. For many communities, the anthropogenic drivers to environmental changes are very opaque, as is the reasoning behind continuing them. For a marginalized community far from the centers of power, the social institutions, inertia, economic cost-benefit analyses, and political considerations which undergird the practices producing a draw-down of aquifers are at least as unknown as how climate change will affect rainfall in the near to medium term, or what their community will look like absent traditional groundwater sources. This is a loss in epistemic self-determination, as the human side of socio-ecological systems becomes much less localized, and intervening in global institutions sometimes requires relying on analyses made by distant others and presented fully formed. Without robust epistemic resources, communities may be left with the options of either accepting the recommendations without understanding its implications (a situation of low epistemic self-determination and a dangerous one to be in for marginalized communities who have historically been exploited by many in positions of epistemic authority), or rejecting the recommendations again without understanding its implications (also a situation of low epistemic self-determination, and also a very dangerous one to be in for marginalized communities).

Some projects which are at least somewhat sensitive to the epistemic self-determination of vulnerable groups on both halves of the socio-ecological framework for adaptation can be found under the umbrellas of the Global Environmental Facility (GEF) Trust Fund/Least Developed Countries Fund (LDCF), and the Special Climate Change Fund (SCCF) which are part of the United Nations Framework Convention on Climate Change (UNFCCC). The GEF/ LDCF and SCCF are funds created in part to help the countries least able to prepare for climate change, which are also quite frequently the ones most likely to be harmed by climate change effects. The most promising set of projects funded by GEF/LDCF and SCCF are CommunityBased Adaptations (CBAs), in which 'The community is considered to be the subject of projects including competence development and techonology transfer to improve adaptive capability.' (Karuk Tribe of California, 2007, p. 6). Though there are certainly dangers of colonization and mis-recognition to be avoided, it is possible that CBAs can increase the capacities of these communities for epistemic self-determination in projects to learn about their environment.

Additionally, these funds have the ability to be affected at the political level by selfdetermined epistemic projects of at least some communities, and for participation in these negotiations to increase those capacities in the future. The funds are monitored and run through the UNFCCC with a Results-Based Management (RBM) approach. This approach has a strategy which includes'Learning and Knowledge Management.'(United Nations Framework Convention on Climate Change, 2009). As an internal document on learning and knowledge management says,

Learning and Knowledge Management is a crucial aspect of RBM for adaptation, in particular the feedback of results in project design and strategy development. ... an objective will be to strengthen knowledge creation, sharing and use-either tacit knowledge that resides with individuals or codified knowledge documented on paper-as a way of doing business.... The LDCF and the SCCF support the implementation of concrete adaptation measures, which may include a wide range of options, such as policy reform and institutional capacity building, adaptation investments in core development sectors, and investment in the field of natural resources management. There is a growing need for lessons and experiences from these types of projects, 
and to ensure that emerging factors influence the GEF's strategies and policies, as well as the projects it finances. (United Nations Framework Convention on Climate Change, 2009, p. 5)

This focus on building capacities is promising, as is the emphasis placed on reciprocity as managers learn about the efficacy of projects from those communities and nations that implement them. This emphasis is not univocal. It is certainly the case that the 'Learning Objectives' and 'Knowledge Products' described in the document use words like 'Dissemination' of knowledge to those countries and communities far more than words like 'Understanding,' which should again make one cautious about reducing epistemic selfdetermination through colonizing practices. Further, some countries have criticized the GEF/ LDCF and the SCCF for being slow, unnecessarily complicated, and opaque (E.g. GEF Evaluation Office, 2011; United Nations Development Programme, 2009). Those criticisms can be understood in our framework here as being in part a response to erosions of selfdetermination, particularly epistemic self-determination. Nevertheless, there is room in the management scheme for some hope that self-determination (including epistemic selfdetermination) for the affected communities will be promoted and expressed in management of the funds.

Further, there was also some space for epistemic self-determination to inform the creation of the funds in the first place. These funds were negotiated between all parties to the UNFCCC, including the least developed countries it seeks to address. To the extent that these countries were representative of or at least informed by the communities within them, self-determined projects by those represented communities were able to affect the funds as they were being created. Indeed, the process of these debates, and participation in the Conferences of the Parties generally, can be a way for representatives from the least developed countries to develop greater understandings of the social, political, and economic causes of climate change as an important element of the Anthropocene, and what the social, political, and economic potentials and hurdles are for addressing these causes. Communities themselves do not participate directly, but this information can filter back to communities of people living within these countries in ways which can be used to increase their epistemic self-determination.

\section{Conclusion}

Much of the damage and loss to communities that are likely outcomes of anthropogenic change, including damage to ecosystems, the loss of land, damage to traditional networks and relationships due to forced migration, and many others besides, have predictable negative impacts on a community's epistemic self-determination. As I have argued, damage to and the potential loss of epistemic self-determination ought itself to be addressed as policy-makers think through loss and damage in general, and epistemic loss in particular along with the loss of an epistemic corpus or suite of methodologies.

I have focused on justice and ethical considerations for promoting communities' epistemic (and other) self-determination in adaptation strategies. In addition to these, there are at least two reasons to think that these kinds of adaptations will make for more resilience (in the sense of an ability of a system to continue despite outside perturbation) (Gallopín, 2006) in the Anthropocene. The first is that epistemologies self-determined by communities are more likely to be responsive to local conditions. As climate change destabilizes weather patterns that have existed throughout human history, affecting flora, fauna, sea levels, and 
a host of other variables, we are only slowly coming to understand we are accelerating toward a period of increasingly less predictable local weather. Additionally, the effect of our civilization on the environment is very irregular; the damage generated by our global culture is not spread equally over the face of the Earth. This fragments our world into often very small environmental regions with their own problems and opportunities. Thus, it becomes increasingly difficult to predict rain in a given year for farmers, but we can predict that those living in quite nearby cities will have markedly higher temperatures than their rural neighbors, and that quite close areas will be affected differently by various pollution sources. Furthermore, pollution and the negative effects of climate change are and will be disproportionately felt by the poor minorities, and other marginalized groups with quite varied means of response. ${ }^{5}$ This unpredictability and local variation points to the benefit of localized, responsive knowledge.

The second reason on practical grounds for preferring epistemic self-determination is that self-determined epistemologies are likely to be quite diverse, and therefore the total system of methodologies is likely to be more resilient. Even if some of the values and methodologies of some epistemologies are not particularly well adapted currently, that may not be sufficient reason to abandon them. Situations can change, sometimes quite drastically. Environmental catastrophe might be the most likely example in our modern world, but various social catastrophes such as war, displacement, economic collapse, and so on all have the possibility of making previously successful practices and values now counterproductive, sometimes to the point of being fatal. At the same time, previously suboptimal approaches may now hold the key to survival. Much like genetic variety in a population suddenly encountering disease or environmental change, epistemic diversity between communities increases the likelihood that more adaptation strategies will survive than in a state of uniformity. Successful strategies can then be adopted with modifications to other contexts in a process of rich epistemic creation, sharing, and adaptation. These practical considerations for increasing communities' epistemic self-determination have borne fruit in the Community-Based Adaptation (CBA) discussed earlier. A recent study (Sherman \& Ford, 2014) of CBA and other community-oriented, 'bottom-up' stakeholder approaches to adaptation in developing nations in comparison with institution-oriented, 'top-down' approaches found that 'Community stakeholder engagement in project design and implementation led to higher effectiveness, efficiency, equity, flexibility, legitimacy, sustainability, and replicability.' (Sherman \& Ford, 2014, p. 418).

It should be pointed out that while epistemic self-determination is important, it is not the only good, and other important considerations may come into conflict with it, such as when the time required to allow a community to fully understand a new policy would result in harmful delays of enacting it. This is true for environmental or public health policies, and could also arise for policies meant to increase epistemic self-determination (e.g. trying to educate communities about climate change when they do not want to learn about it and even deny its existence, though that knowledge would allow them to better understand what is happening around them). In such cases of conflict, difficult decisions are necessary, though again those decisions can be made through deliberations that try to maximize self-determination. The effect on epistemic self-determination of various adaptation policies is an important standard for evaluating different strategies of cooperation and coordination between communities and between communities and larger social institutions. Cultivating fertile epistemic sharing among communities and encouraging transparency and 
accountability in more powerful groups are much more desirable by this standard than strategies of centralization. Though many of the environmental changes on the horizon from anthropogenic drivers are ineluctable in the near to middle term, our responses to them are much less fixed. In the case of social adaptation in particular, we can adapt in ways which are more likely to be effective or more likely to be ineffective, and more or less supportive of individual and community flourishing. The context of communities' epistemic self-determination at least is one example of more just, localized, and anti-hierarchical social institutions being preferable on both practical and ethical grounds.

\section{Notes}

1. I would be remiss at this point not to say that as of this writing, it seems that the U.S. Government is moving in a direction of being even less open to these arguments than they have been previously. This could have devastating consequences for the success of any fund to compensate and aid adaptation for poor and affected countries.

2. For a discussion of WIM, see Mathew and Akter (2015).

3. For a longer discussion of this term, see Werkheiser (2016).

4. e.g. the Native American communities discussed in Arquette et al. (2002).

5. For how these differences play out for urban areas, see Stone (2012).

\section{Disclosure statement}

No potential conflict of interest was reported by the author.

\section{References}

Alessa, L., Kliskey, A., \& Williams, P. (2010). Forgetting freshwater: Technology, values, and distancing in remote arctic communities. Society and Natural Resources, 23, 254-268.

Arquette, M., Cole, M., Cook, K., LaFrance, B., Peters, M., Ransom, J., ... Stairs, A. (2002). Holistic riskbased environmental decision making: A native perspective. Environmental Health Perspectives, 110, 259-264.

Baatz, C. (2013). Responsibility for the past? Some thoughts on compensating those vulnerable to climate change in developing countries. Ethics, Policy \& Environment, 16, 94-110.

Biermann, F., \& Boas, I. (2010). Preparing for a warmer world: Towards a global governance system to protect climate refugees. Global Environmental Politics, 10, 60-88.

Carter, I. (1999). A measure of freedom. Oxford: Oxford University Press.

Chief, K., Daigle, J. J., Lynn, K., \& Whyte, K. (2014). Indigenous experiences in the US with climate change and environmental stewardship in the anthropocene. USDA Forest Service RMRS-P-71.

Evans, P. (2002). Collective capabilities, culture, and Amartya Sen's development as freedom. Studies in Comparative International Development, 37, 54-60.

Figueroa, R. (2006). Evaluating environmental justice claims. In J. Bauer (Ed.), Forging environmentalism: Justice, livelihood, and contested environments (pp. 360-376). Armonk, NY: M.E. Sharpe.

Gallopín, G. C. (2006). Linkages between vulnerability, resilience, and adaptive capacity. Global Environmental Change, 16, 293-303.

GEF Evaluation Office. (2011). Evaluation of the special climate change fund (SCCF) (GEF/LDCF.SCCF.11/ $\mathrm{ME} / 02$ ).

Gould, C. C. (2014). Interactive democracy: The social roots of global justice. Cambridge: Cambridge University Press.

Ibrahim, S. S. (2006). From individual to collective capabilities: The capability approach as a conceptual framework for self-help. Journal of Human Development, 7, 397-416.

Karuk Tribe of California. (n.d.). Bring the salmon home: The Karuk tribe efforts to remove Klamath dams. Press release. Retrieved from https://www.karuk.us/press 
Karuk Tribe of California. (2006). Karuk tribe department of natural resources eco-cultural resources management plan. Orleans, CA: Karuk Tribe Department of Natural Resources.

Karuk Tribe of California. (2007). Water quality assessment report: Klamath River, Salmon River, Scott River, Shasta River, Ti-Bar Creek, and Irving Creek. Orleans, CA: Department of Natural Resources.

Mathew, L. M., \& Akter, S. (2015). Loss and damage associated with climate change impacts. In W.-Y. Chen, T. Suzuki, \& M. Lackner (Eds.), Handbook of climate change mitigation and adaptation (pp. 17-45). New York, NY: Springer.

Mendelsohn, R., Dinar, A., \&Williams, L. (2006). The distributional impact of climate change on rich and poor countries. Environment and Development Economics, 11, 159-178.

Menser, M. (2008). Transnational participatory democracy in action: The case of La via Campesina. Journal of Social Philosophy, 39, 20-41.

Norgaard, K. (2004). The effects of altered diet on the health of the Karuk people: A preliminary report. White paper. Written under contract by the Karuk Tribe of California Department of Natural Resources Water Quality Program.

Norton, B. G. (2005). The rebirth of environmentalism as pragmatic, adaptive management. Virginia Environmental Law Journal, 24, 353-384.

Pettit, P. (2001). Deliberative democracy and the discursive dilemma. Philosophical Issues, 11 (supplement to Nous), 268-299.

Pierce, R. M. (1998). Klamath Salmon: Understanding allocation - background, policy of the procedures of the harvest allocation process for Klamath River fall Chinook salmon. White paper. Prepared for the Klamath River Basin Fisheries Task Force United States Fish and Wildlive Services.

Reed, R., \& Norgaard, K. (2010). Salmon fees our people: Challenging dams on the Klamath River. In K. Walker Painemilla, A. B. Rylands, A. Woofter, \& C. Hughes (Eds.), Indigenous peoples and conservation: From rights to resource management (1st ed., pp. 7-17). Arlington, VA: Conservation International.

Reed, R., Sarna, D., Diver, S., \& Lynn, K. (2012). Preserving tribal self-determination and knowledge sovereignty while expanding use of tribal knowledge and management in off reservation lands in the face of climate change. North Pacific Landscape Conservation Cooperative.

Sherman, M. H., \& Ford, J. (2014). Stakeholder engagement in adaptation interventions: An evaluation of projects in developing nations. Climate Policy, 14, 417-441.

Shrader-Frechette, K. (1991). Risk and rationality: Philosophical foundations for populist reforms. Berkeley, CA: California University Press.

Stone Jr., B. (2012). The city and the coming climate: Climate change in the places we live. New York, NY: Cambridge University Press.

Taylor, D. (2000). The rise of the environmental justice paradigm: Injustice framing and the social construction of environmental discourses. American Behavioral Scientist, 43, 508-580.

United Nations Development Programme. (2009). Evaluation of UDNP work with least developed countries fund and special climate change fund resources. UNDP Evaluations Office.

United Nations Framework Convention on Climate Change. (2009). Implementation of results-based management under the least developed countries fund and the special climate change fund (GEF/LDCF. SCCF.7/4).

United Nations Framework Convention on Climate Change. (2013). Non-economic losses in the context of the work programme on loss and damage (Report No. GE.13-63820).

Voggesser, G., Lynn, K., Daigle, J., Lake, F. K., \& Ranco, D. (2013). Cultural impacts to tribes from climate change influences on forests. Climatic Change, 120, 615-626.

Wildcat, D. (2013). Introduction: Climate change and indigenous peoples of the USA. Climatic Change, 120, 509-515.

Werkhseiser, I. (2014). Food sovereignty, health sovereignty, and self-organised community viability. Interdisciplinary Environmental Review, 15, 134-146.

Werkheiser, I. (2016). Food policies empowering democratic and epistemic self-determination. Journal of Social Philosophy, 47, 25-40.

Werkheiser, I. (2015). Community epistemic capacity. Social Epistemology: A Journal of Knowledge, Culture, and Policy, 30, 25-44.

Whyte, K. (in press). What do Indigenous knowledges do for Indigenous peoples?

Whyte, K. (2017, January/February). The Dakota access pipeline, environmental injustice, and U.S. colonialism. Forthcoming in Red Ink: An International Journal of Indigenous Literature, Arts, \& Humanities. Retrieved from SSRN: https://ssrn.com/abstract=2925513 